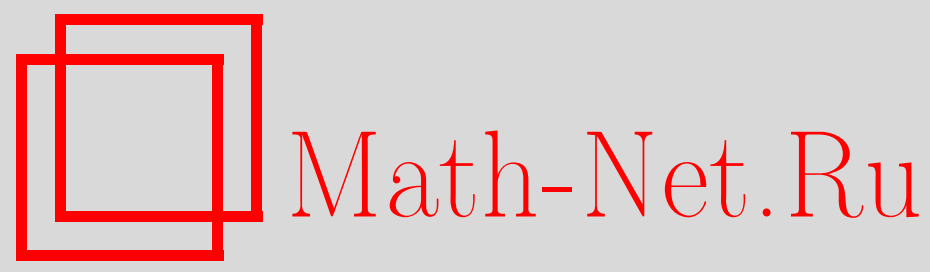

А. Ю. Веснин, А. Д. Медных, Сферические группы Коксетера и гиперэллиптические 3-многообразия, Матем. заметки, 1999, том 66, выпуск 2, 173-177

DOI: https://doi.org/10.4213/mzm1152

Использование Общероссийского математического портала Math-Net.Ru подразумевает, что вы прочитали и согласны с пользовательским соглашением http://www . mathnet.ru/rus/agreement

Параметры загрузки:

IP: 34.229 .45 .116

26 апреля 2023 г., 10:16:49

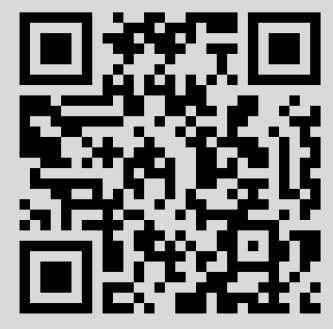




\title{
СФЕРИЧЕСКИЕ ГРУППЫ КОКСЕТЕРА И ГИПЕРЭЛЛИПТИЧЕСКИЕ 3-МНОГООБРАЗИЯ
}

\author{
А. Ю. Веснин, А. Д. Медных
}

Изучаются группы отражений коксетеровских полиэдров в трехмерных геометриях Терстона. Для широкого класса групп Коксетера устанавливается существование подгрупп конечного индекса, униформизирующих трехмерные гиперэллиптические многообразия.

Библиографиял: 7 названий.

Пусть $X^{3}$ - одна из трехмерных геометрий: $\mathbb{H}^{3}, \mathbb{E}^{3}, \mathbb{S}^{3}, \mathbb{H}^{2} \times \mathbb{E}^{1}$ или $\mathbb{S}^{2} \times \mathbb{E}^{1}[1]$. В работе рассматриваются трехмерные многообразия $M^{3}$, возникающие как факторпространства $M^{3}=X^{3} / \Gamma$, где $\Gamma$ - дискретная группа изометрий, действуюшая на $X^{3}$ без неподвижных точек. Трехмерное многообразие $M^{3}$ называется гиперәллиптическим, если сушествует изометрическая инволюция $\tau$ такая, что факторпространство $M^{3} /\langle\tau\rangle$ гомеоморфно трехмерной сфере $\mathscr{S}^{3}$. Существование гиперэллиптических многообразий для каждой из трехмерных геометрий было установлено в [2]. При этом ключевую роль при построении гиперэллиптических многообразий играют прямоугольные полиэдры, обладающие гамильтоновыми циклами. В данной работе рассматривается более широкий класс коксетеровских полиэдров и устанавливается их связь с гиперэллиптическими многообразиями.

Пусть $P$ - коксетеровский полиэдр (т.е. полиэдр с двугранными углами вида $\pi / n$, где целое $n \geqslant 2$ ) в одной из перечисленных вьше трехмерных геометрий. Его скелетом $P^{1}$ будем назьвать взвешенньй граф, вершины и ребра которого совпадают соответственно с вершинами и ребрами полиэдра $P$. При этом вес ребра $e \in P^{1}$ равен $n$, если двугранный угол при соответствующем ребре полиэдра $P$ равен $\pi / n$.

Напомним [3], что граф $H$ назьвается остовным подграфом графа $G$, если множества их вершин совпадают, а множество ребер графа $H$ является подмножеством множества ребер графа $G$.

Два взвешенных графа $G$ и $H$ с целыми положительными весами будем называть гомеоморфными, если они могут быть получены из одного и того же взвешенного графа подразбиением его ребер веса 2.

Напомним некоторые факты из теории коксетеровских групп [4]. Пусть $p \subset \mathbb{S}^{3}-$ коксетеровский полиэдр, а $\delta(p)$ - группа, порожденная отражениями в его гранях. Отметим, что все групшы $\delta(p)$ конечны и полностью классифицированы. Схемы груп Кок-

Работа выполнена при поддержке Российского фонда фундаментальных исследований, грант № 98-01-00699. 

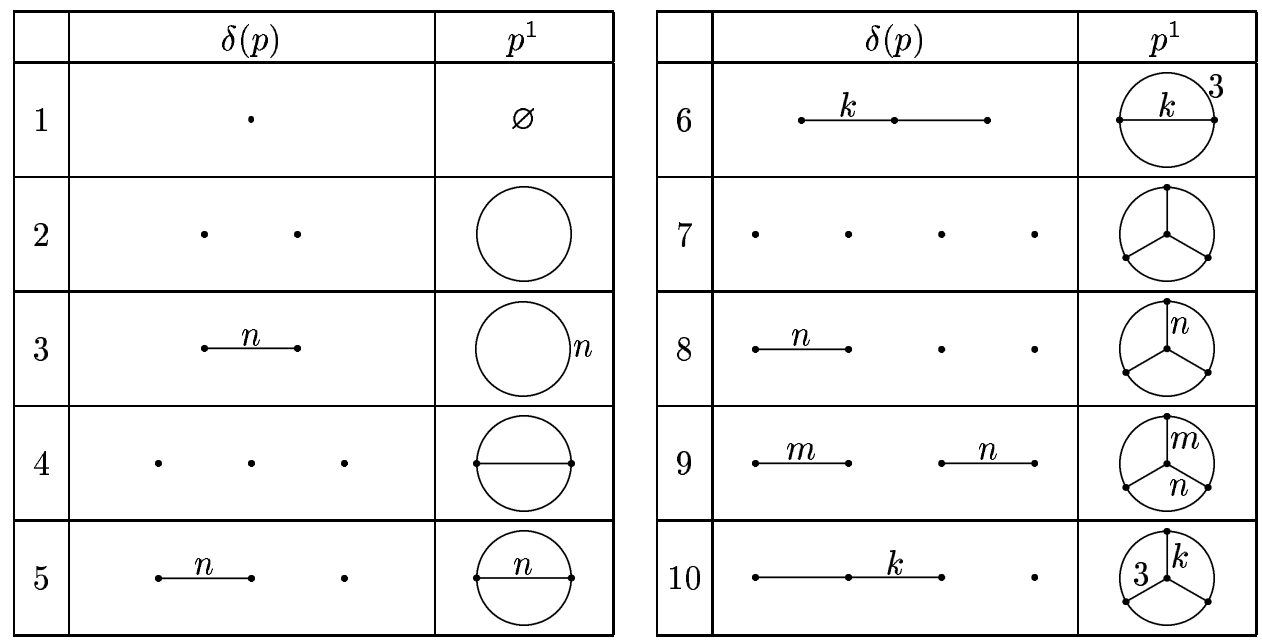

\section{ТАБЛИЦА 1}

сетера $\delta(p)$ и скелетов $p^{1}$, соответствующих трехмерньм сферическим коксетеровским полиэдрам $p$, приведены в табл. 1 .

Для упрощения обозначений при изображении взвешенного графа $p^{1}$ мы опускаем метку 2 на его ребрах. Указанные в табл. 1 целые параметры удовлетворяют условиям $m, n \geqslant 3$ и $k=3,4,5$.

Для заданного сферического коксетеровского полиэдра $p$ со скелетом $p^{1} \neq \varnothing$ мы будем рассматривать коксетеровские полиэдры $P$ в пространствах $X^{3}$, скелеты $P^{1}$ которых содержат остовные подграфы, гомеоморфные скелету $p^{1}$. В каждом случае класс таких многогранников достаточно широк. В частности, в случае 2 табл. 1 полиэдру $p$ соответствуют прямоугольные гамильтоновы полиэдры $P$.

Следующая теорема устанавливает существование подгрушы конечного индекса в группе, порожденной отражениями в гранях полиэдра $P$, которая униформизирует гиперэллиптическое многообразие.

ТЕОрема. Пусть $p$ - коксетеровский полиэдр в $\mathbb{S}^{3}$ и $P$ - коксетеровский полиәдр в $X^{3}$, әде $X^{3}=\mathbb{H}^{3}, \mathbb{E}^{3}, \mathbb{S}^{3}, \mathbb{S}^{2} \times \mathbb{E}^{1}$ или $\mathbb{H}^{2} \times \mathbb{E}^{1}$, а $\delta(p)$ и $\Delta(P)$ - соответствующие им группы отражсний. Предположим, что скелет $P^{1}$ полиэдра $P$ содержит собственный остовный подграф $T$, гомеоморфный скелету $p^{1}$ полиәдра $p$, и все ребра $P^{1} \backslash T$ имеют вес 2 . Тогда в группе $\Delta(P)$ существует подгруппа $G$ индекса $|\Delta(P): G|=2|\delta(p)|$ без кручения такая, что $M^{3}=X^{3} / G$ - гиперәллиптическое многообразие.

ДоКАЗАТЕЛЬСТво. Прежде всего отметим, что в дальнейшем мы будем использовать терминологию и основные факты теории трехмерных орбифолдов [5, гл. 13].

Пусть $\mathscr{O}_{1}$ и $\mathscr{O}_{2}$ - два орбифолда с универсальным накрывающим пространством $\mathscr{O}$ и орбифолдными фундаментальными группами $\Gamma_{1}$ и $\Gamma_{2}$ соответственно. При этом имеют место канонические накрытия $\pi_{1}: \mathscr{O} \rightarrow \mathscr{O}_{1}=\mathscr{O} / \Gamma_{1}$ и $\pi_{2}: \mathscr{O} \rightarrow \mathscr{O}_{2}=\mathscr{O} / \Gamma_{2}$. Пусть $\Gamma_{1} \subset \Gamma_{2}$ и $\pi: \mathscr{O}_{1} \rightarrow \mathscr{O}_{2}$ - орбифолдное накрытие, индуцированное групповым включением. Порядком ветвления накрытия $\pi$ в точке $x \in \mathscr{O}_{1}$ будем назьвать величину $\left|\left(\Gamma_{2}\right)_{\widetilde{x}}:\left(\Gamma_{1}\right)_{\widetilde{x}}\right|$, где $\widetilde{x}=\pi_{1}^{-1}(x)$, a $\left(\Gamma_{i}\right)_{\widetilde{x}}$-стабилизатор групы $\Gamma_{i}$ в точке $\widetilde{x}$. В частности, 
если накрытие $\pi$ неразветвлено в точке $x \in \mathscr{O}_{1}$, то $\left|\left(\Gamma_{2}\right)_{\tilde{x}}:\left(\Gamma_{1}\right)_{\tilde{x}}\right|=1$ и $\left(\Gamma_{1}\right)_{\tilde{x}}=\left(\Gamma_{2}\right)_{\tilde{x}}$.

Пусть $X, Y$ - метрические пространства. Для накрытия $\pi: X \rightarrow Y$ определим группу $\operatorname{Cov}_{\pi}(X, Y)=\{h \in \operatorname{Homeo}(X): \pi \circ h=\pi\}$. Накрытие $\pi$ будем назьвать $р$ егулярным $G$-накрытием, разветвленным над взвешенным графом $T \subset Y$, если выполнены следуюшие условия:

a) $\operatorname{Cov}_{\pi}(X, Y) \cong G$

б) отображение $\bar{\pi}: X \backslash \pi^{-1}(T) \rightarrow Y \backslash T$, индуцированное отображением $\pi$, является неразветвленным накрытием;

в) факторпространство $X / \operatorname{Cov}_{\pi}(X, Y)$ является орбифолдом с носителем $Y$ и сингулярньп множеством $T$.

Два накрытия $\pi: X \rightarrow Y$ и $\pi^{\prime}: X^{\prime} \rightarrow Y$ будем называть әквивалентными, если существует гомеоморфизм $h: X \rightarrow X^{\prime}$ такой, что $\pi=\pi^{\prime} \circ h$.

Пусть $\mathscr{O}_{1}$ и $\mathscr{O}_{2}$ - орбифолды с носителями $X$ и $Y$ соответственно, а $T$ - взвешенньй граф в $Y$. Орбифолдное накрытие $\pi: \mathscr{O}_{1} \rightarrow \mathscr{O}_{2}$ будем назьвать регулярным $G$-накрытием, разветвленным над $T$, если индуцированное им накрытие носителей $\pi_{X}: X \rightarrow$ $Y$ удовлетворяет условиям а)-в).

Итак, обозначим через $\delta^{+}(p)$ и $\Delta^{+}(P)$ подгрупшы индекса 2 в группах $\delta(p)$ и $\Delta(P)$, состоящие из всех сохраняюших ориентацию изометрий. Тогда $\Omega^{3}=X^{3} / \Delta^{+}(P)$ - орбифолд, носителем которого является трехмерная сфера $\mathscr{S}^{3}$, а сингулярньм множествомвзвешенньй граф $P^{1}$. Аналогично, $\omega^{3}=\mathbb{S}^{3} / \delta^{+}(p)$ - орбифолд, носителем которого является $\mathscr{S}^{3}$, а сингулярным множеством - взвешенный граф $p^{1}$.

ЛЕмма 1. Пусть $p$ - сферический коксетеровский полиәдр, а $p^{1} \subset \mathscr{S}^{3}-$ его скелет. Тогда с точностью до әквивалентности существует единственное регулярное $\delta^{+}(p)$-накрытие $\pi: \Sigma^{3} \rightarrow \mathscr{S}^{3}$, разветвленное над $p^{1}$. При этом $\Sigma^{3}$ гомеоморфно $\mathscr{S}^{3}$.

ДокАЗАТЕЛЬСтво. Отметим, что универсальное накрытие

$$
\pi_{p}: \mathscr{S}^{3} \rightarrow \omega^{3}=\mathbb{S}^{3} / \delta^{+}(p)
$$

удовлетворяет условиям леммы. Предположим, что существует отличное от него регулярное $\delta^{+}(p)$-накрытие $\pi: \Sigma^{3} \rightarrow \mathscr{S}^{3}$, разветвленное над $p^{1}$. Покажем, что накрытия $\pi_{p}$ и $\pi$ эквивалентны. В самом деле, эти накрытия индуцируют неразветвленные $\delta^{+}(p)$-накрытия $\bar{\pi}_{p}$ и $\bar{\pi}$ такие, что $\bar{\pi}_{p}: \mathscr{S}^{3} \backslash \pi_{p}^{-1}\left(p^{1}\right) \rightarrow \mathscr{S}^{3} \backslash p^{1}$ и $\bar{\pi}: \Sigma^{3} \backslash \pi^{-1}\left(p^{1}\right) \rightarrow \mathscr{S}^{3} \backslash p^{1}$.

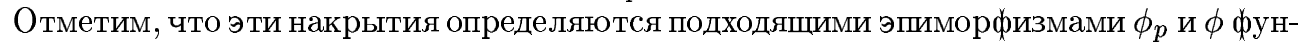
даментальной группы $\Gamma=\pi_{1}\left(\mathscr{S}^{3} \backslash p^{1}\right)$ на группу $\delta^{+}(p)$. При этом $\pi_{1}\left(\mathscr{S}^{3} \backslash \pi_{p}^{-1}\left(p^{1}\right)\right)=$ $\operatorname{Ker} \phi_{p}$ и $\pi_{1}\left(\Sigma^{3} \backslash \pi^{-1}\left(p^{1}\right)\right)=\operatorname{Ker} \phi$. Поскольку накрытия $\pi_{p}$ и $\pi$ разветвлены над $p^{1}$, эпиморфизмы $\phi_{p}$ и $\phi$ обладают следуюшим свойством: если $\gamma_{e}$-однократная петля вокруг ребра $е$ граффа $p^{1}$ с весом $n_{e}$, то порядки элементов $\phi_{p}\left(\gamma_{e}\right)$ и $\phi\left(\gamma_{e}\right)$ в групше $\delta^{+}(p)$ равны $n_{e}$.

Для множества $A=\left\{\gamma_{e}^{n_{e}} \mid e \in p^{1}\right\}$ обозначим через $\operatorname{Ncl}(A)$ его нормальное замыкание в группе Г и рассмотрим канонический гомоморфизм

$$
\varphi: \Gamma \rightarrow \Gamma / \operatorname{Ncl}(A)
$$

В силу отмеченных выше свойств эпиморфизмов $\phi_{p}$ и $\phi$ существуют эпиморфизмы $\theta_{p}: \Gamma / \operatorname{Ncl}(A) \rightarrow \delta^{+}(p)$ и $\theta: \Gamma / \operatorname{Ncl}(A) \rightarrow \delta^{+}(p)$ такие, что $\phi_{p}=\theta_{p}$ о $\varphi$ и $\phi=\theta$ о $\varphi$. 
В силу [6] групша $\Gamma / \operatorname{Ncl}(A)$ является группой орбифолда с носителем $\mathscr{S}^{3}$, сингулярным множеством которого является взвешенньй граф $p^{1}$, т.е. орбифолда $\omega^{3}$. Таким обpaзом, $\Gamma / \operatorname{Ncl}(A) \cong \delta^{+}(p)$, а $\theta_{p}$ и $\theta$ являются эпиморфизмами группы $\delta^{+}(p)$ на себя. Поскольку группа сферических изометрий $\delta^{+}(p)$ конечна, $\theta_{p}$ и $\theta$ являются изоморфизмами. Таким образом, эпиморфизмы $\phi_{p}$ и $\phi$ отличаются на некоторьй автоморфизм $\alpha$ группы $\delta^{+}(p)$, т.е. $\phi_{p}=\alpha \circ \phi$. Значит, ядра $\operatorname{Ker} \phi_{p}$ и $\operatorname{Ker} \phi$ совпадают, и накрытия $\bar{\pi}_{p}$ и $\bar{\pi}$ эквивалентны.

По теореме $Ф$ окса [7] каждое из накрытий $\bar{\pi}_{p}$ и $\bar{\pi}$ допускает единственное, с точностью до эквивалентности, пополнение до регулярного $\delta^{+}(p)$-накрытия, разветвленного над $p^{1}$. Следовательно, накрытия $\pi_{p}$ и $\pi$ эквивалентны, что завершает доказательство леммы.

Следуя [2], ориентируемьй орбифолд $\mathscr{O}$ будем называть зиперәллиптической сферой, если его носителем является трехмерная сффера $\mathscr{S}^{3}$, а сингулярным множеством зацепление (в частности, узел) в $\mathscr{S}^{3}$, индекс сингулярности каждой компоненты которого равен 2 .

Рассмотрим собственньй остовный подграф $T$ графа $P^{1}$, гомеоморфньй графу $p^{1}$.

Лемма 2. Пусть $\pi: \Sigma^{3} \rightarrow \Omega^{3}$ - регулярное орбифолдное $\delta^{+}(p)$-накрытие, разветвленное над T. Тогда $\Sigma^{3}$ - гиперэллиптическая сфера.

ДокАЗАтЕЛЬство. Положим $\Gamma_{0}=\Delta^{+}(P)$. Тогда $\Omega^{3}=X^{3} / \Gamma_{0}$. Представим $\Sigma^{3}$ в виде $\Sigma^{3}=X^{3} / \Gamma$, где $\Gamma \triangleleft \Gamma_{0}$ и $\Gamma_{0} / \Gamma \cong \delta^{+}(p)$. Для канонических накрытий $\sigma: X^{3} \rightarrow \Sigma^{3}=$ $X^{3} / \Gamma$ и $\omega: X^{3} \rightarrow \Omega^{3}=X^{3} / \Gamma_{0}$ рассмотрим произвольную точку $z \in \Sigma^{3}$ и ее прообраз $\tilde{z} \in \sigma^{-1}(z)$. Положим $z^{\prime}=\omega(\tilde{z})$.

Для достаточно малого шара $V_{\tilde{z}}=B(\tilde{z}, \varepsilon)$ орбифолдное накрытие $\pi: \Sigma^{3} \rightarrow \Omega^{3}$ локально имеет вид $\pi: V_{\tilde{z}} / \Gamma_{\tilde{z}} \rightarrow V_{\tilde{z}} /\left(\Gamma_{0}\right)_{\tilde{z}}$, где $\Gamma_{\tilde{z}}$ и $\left(\Gamma_{0}\right)_{\tilde{z}}-$ стабилизаторы точки $\tilde{z}$ в группах $Г$ и $\Gamma_{0}$ соответственно. В частности, порядок ветвления накрытия $\pi$ в точке $z$ равен $\left|\left(\Gamma_{0}\right)_{\tilde{z}}: \Gamma_{\tilde{z}}\right|$.

Для точки $z^{\prime} \in \Omega^{3}$ имеет место одна из следующих пяти возможностей:

a) $z^{\prime} \notin P^{1}$;

б) $z^{\prime}$ - внутренняя точка ребра $e \in P^{1} \backslash T$;

в) $z^{\prime}$ - внутренняя точка ребра графа $T$;

г) $z^{\prime}$ - двухвалентная вершина графа $T$;

д) $z^{\prime}$ - тривалентная вершина графа $T$.

Изучим строение стабилизаторов $\left(\Gamma_{0}\right) \tilde{z}$ и $\Gamma_{\tilde{z}}$ в каждом из этих случаев. В случае а) имеем $\Gamma_{\tilde{z}}=\left(\Gamma_{0}\right)_{\tilde{z}}=\langle 1\rangle$. В случае б) поскольку все ребра $P^{1} \backslash T$ имеют вес 2 , двугранный угол при ребре $e$ равен $\pi / 2$. Накрытие $\pi: \Sigma^{3} \rightarrow \Omega^{3}$ неразветвлено над $e$, поэтому $\Gamma_{\tilde{z}}=\left(\Gamma_{0}\right)_{\tilde{z}} \cong \mathbb{Z}_{2}$. В случае в) рассмотрим ребро е графа $T$, для которого точка $z^{\prime}$ является внутренней. Пусть двугранньй угол при ребре $e$ равен $\pi / n, n \geqslant 2$. Тогда $\left(\Gamma_{0}\right)_{\tilde{z}} \cong \mathbb{Z}_{n}$. Поскольку $\pi$ является $\delta^{+}(p)$-накрытием, разветвленньгм над $T$, имеем $\left|\left(\Gamma_{0}\right)_{\tilde{z}}: \Gamma_{\tilde{z}}\right|=n$. Следовательно, $\Gamma_{\tilde{z}} \cong\langle 1\rangle$. В случае г) точка $z^{\prime}$ является вершиной графа $T$, и ровно два из трех инцидентных ей ребер принадлежат $T$. Поскольку $T$ является остовным подграфом скелета $P^{1}$, гомеоморфньм $p^{1}$, для всех трех ребер, инцидентных $z^{\prime}$, индекс сингулярности равен 2 . Следовательно, $\left(\Gamma_{0}\right)_{z} \cong \mathbb{Z}_{2} \oplus \mathbb{Z}_{2}$. Поскольку накрытие $\pi$ разветвлено над точкой $z^{\prime} \in T$, то $\left|\left(\Gamma_{0}\right)_{\tilde{z}}: \Gamma_{\tilde{z}}\right|=2 ;$ следовательно, $\Gamma_{\tilde{z}} \cong \mathbb{Z}_{2}$. В случае д) накрытие $\pi$ в точке $z$ имеет порядок ветвления $\left|\left(\Gamma_{0}\right)_{\tilde{z}}: \Gamma_{\tilde{z}}\right|$, равньй порядку группы $\left(\Gamma_{0}\right)_{\tilde{z}}$, откуда $\Gamma_{\tilde{z}}=\langle 1\rangle$. 
Таким образом, во всех рассмотренных случаях либо $\Gamma_{\tilde{z}}=\langle 1\rangle$ и $z$ не является сингулярной точкой, либо $\Gamma_{\tilde{z}} \cong \mathbb{Z}_{2}$ и $z$ является сингулярной точкой с индексом сингулярности 2. Это означает, что локально сингулярное множество орбифолда $\Sigma^{3}$ гомеоморфно открытому интервалу с индексом 2 . По лемме 1 носитель орбифолда $\Sigma^{3}$ гомеоморфен $\mathscr{S}^{3}$. Таким образом, орбифолд $\Sigma^{3}$ является гиперэллиптической сферой. Лемма доказана.

Продолжим доказательство теоремы. Рассмотрим регулярное $\delta^{+}(p)$-накрытие

$$
\pi: \Sigma^{3}=X^{3} / \Gamma \rightarrow \Omega^{3}=X^{3} / \Delta^{+}(P),
$$

разветвленное над $T$. В силу леммы 2 орбифолд $\Sigma^{3}$ является гиперэллиптической сферой, униформизируемой группой $\Gamma \triangleleft \Delta^{+}(P)$. Как показано в [2], если $\Sigma^{3}-$ гиперэллиптическая сфера, то существует гиперэллиптическое многообразие $M^{3}$ с инволюцией $\tau$ такое, что $M^{3} /\langle\tau\rangle=\Sigma^{3}$. Накрытие $M^{3} \rightarrow \Sigma^{3}$ индуцирует групповое включение $G \triangleleft \Gamma$, где $G=\pi_{1}\left(M^{3}\right)$ - подгруппа индекса 2 в группе $\Gamma$, не содержащая элементов конечного порядка. Как нетрудно видеть, индекс подгрупшы $G$ в группе $\Delta(P)$ равен $|\Delta(P): G|=2|\delta(p)|$. Теорема доказана.

Отметим, что частньй случай теоремы для гамильтоновых прямоугольных полиэдров (случай 2 табл. 1) был установлен ранее в [2].

\section{СПИСОК ЦИТИРОВАННОЙ ЛИТЕРАТУРЫ}

[1] Scott P. The geometries of 3-manifolds // Bull. London Math. Soc. 1986. V. 15. P. 401-487.

[2] Mednykh A.D. Three-dimensional hyperelliptic manifolds // Ann. Global Anal. Geom. 1990. V. 8. P. 13-19.

[3] Harary F. Graph Theory. London: Addison-Wesley Publ., 1969.

[4] Coxeter H. M.S. Discrete groups generated by reflections // Ann. Math. 1934. V. 35. P. 588-621.

[5] Thurston W. The Geometry and Topology of 3-Manifolds. Lecture Notes. Princeton: Princeton Univ. Press, 1980.

[6] Haefliger A., Quach N.D. Une présentation de groupe fundamental d'une orbifold // Asterisque. 1984. V. 116. P. 98-107.

[7] Fox R.H. Covering spaces with singularities // Algebraic Geometry and Topology / ed. R.H. Fox, D. C. Spencer, A. W. Tucker. Princeton: Princeton Univ. Press, 1957. P. 243-257.

Институт математики им. С. Л. Соболева СО РАН, г. Новосибирск 